\title{
Human Immunodeficiency Virus Type 1 Alters Brain- Derived Neurotrophic Factor Processing in Neurons
}

\author{
Alessia Bachis, ${ }^{1}$ Valeriya Avdoshina, ${ }^{1}$ Luigi Zecca, ${ }^{2}$ Maia Parsadanian, ${ }^{1}$ and Italo Mocchetti ${ }^{1}$ \\ ${ }^{1}$ Department of Neuroscience, Georgetown University Medical Center, Washington, DC 20057 and ${ }^{2}$ CNR Institute of Biomedical Technologies, 20090 \\ Segrate, Italy
}

The molecular mechanisms leading to synaptic simplification and neuronal apoptosis in human immunodeficiency virus type 1 (HIV1)-positive subjects are unknown. The HIV protein gp120 reduced the length of neuronal processes similarly to the proneurotrophin pro- brain-derived neurotrophic factor (proBDNF). Intriguingly, the effects of both proBDNF and gp 120 were blocked by inhibitors of the p75 neurotrophin receptor, suggesting that proBDNF and gp120 share a similar mechanism of neurotoxicity. Therefore, we tested the hypothesis that gp120 affects the release of proBDNF. Using rat primary neurons, we observed that gp120 promotes a time-dependent intracellular and extracellular accumulation of proBDNF concomitantly with a decrease in mature BDNF. A similar imbalance in the ratio proBDNF/mature BDNF was confirmed in postmortem brains of HIV-positive subjects cognitively impaired and motor impaired. Therefore, it is conceivable to formulate the hypothesis that HIV neurotoxicity includes a gp120-mediated alteration of BDNF processing. To determine the cellular mechanism whereby gp120 produces an accumulation of proBDNF, we examined the levels of intracellular and extracellular enzymes that proteolytically cleave proBDNF furin and tissue plasminogen, respectively. In rat neurons exposed to gp120, intracellular furin levels decreased before cell death, whereas tissue plasminogen changed only during apoptosis. Our data suggest that HIV, through gp 120, reduces proBDNF processing by affecting furin levels, and therefore causes an altered balance between antiapoptotic and proapoptotic neurotrophins. Our studies identify a new mechanism that may explain how HIV promotes neuronal injury.

\section{Introduction}

Various degrees of synaptic pruning and neuronal apoptosis are seen in human immunodeficiency virus type 1 (HIV-1)-positive subjects in the late stage of infection of their brain (Ellis et al., 2007). These abnormalities culminate in neurocognitive deficits termed HIV-associated neurocognitive disorders, and especially in their more severe form, or HIV-associated dementia (HAD). However, the molecular mechanisms leading to these neuropathological features remain unknown.

Much effort is being devoted to understanding how HIV promotes neuronal degeneration and the role played by immune activation (Gartner and Liu, 2002) combined with the effects of host cell-derived factors (Kaul et al., 2001) and viral proteins (Kerr et al., 1997). Nevertheless, atrophy of axons and neuronal processes often precedes the death of the cell body. Moreover, synaptodendritic injury is a form of degeneration that occurs in other neurodegenerative diseases that do not exhibit immune response, such as Alzheimer's disease (Masliah, 1995) or neu-

Received Feb. 22, 2012; revised May 2, 2012; accepted May 8, 2012.

Author contributions: A.B., V.A., and M.P. performed research; L.Z. contributed unpublished reagents/analytic tools; A.B. analyzed data; I.M. wrote the paper.

This work was supported by Health and Human Services Grants 1R01DA026174 and U01MH083501 and the Latham Trust Fund. L.Z. was supported by grant RBNE08ZZN7 from the Italian Ministry of University. We thank the National NeuroAIDS Tissue Consortium and the Section of Legal Medicine and Insurances of the University of Milan (Milan, Italy).

Correspondence should be addressed to Italo Mocchetti, Georgetown University Medical Center, WP13 New Research Building, 3970 Reservoir Road, Northwest, Washington DC 20057. E-mail: moccheti@georgetown.edu.

DOI:10.1523/JNEUROSCI.0865-12.2012

Copyright $\odot 2012$ the authors $\quad 0270-6474 / 12 / 329477-08 \$ 15.00 / 0$ rotrophic factor withdrawal (Raff et al., 2002). Intriguingly, both HIV (Avdoshina et al., 2011) and its envelope protein gp120 (Nosheny et al., 2004) have been shown to reduce the levels of brain-derived neurotrophic factor (BDNF), a potent prosurvival neurotrophic factor that plays a role in synaptic plasticity including modulation of dendritic branching and spine morphology (Horch, 2004; Tanaka et al., 2008) and neurogenesis (Li et al., 2008). Thus, it is plausible to suggest that HIV may promote synaptodendritic degeneration by multiple mechanisms, which include a reduction of relevant neurotrophic factors. This suggestion would be in line with the theory that reduced expression of BDNF is involved in neurodegenerative diseases (Zuccato et al., 2001). Discovering how HIV reduces BDNF should help clinicians identify new adjunct therapies.

Mature BDNF (mBDNF) is synthesized as a larger glycosylated precursor, proBDNF (Mowla et al., 2001), which can be released from neurons (Yang et al., 2009b). When proBDNF is not cleaved, it mediates biological effects that are opposite of those of mBDNF, including neuronal apoptosis (Teng et al., 2005) and presynaptic terminal retraction (Yang et al., 2009a). These events are initiated by proBDNF binding to the lowaffinity p75 neurotrophin receptor (p75NTR) in concert with the coreceptor sortilin (Teng et al., 2005). Thus, neuronal survival/ death depends on whether proBDNF rather than mBDNF is released. ProBDNF is proteolitically processed to mBDNF by plasmin or metalloproteases (Pang et al., 2004). HIV has been shown to alter metalloproteases within the CNS (Conant et al., 1999); therefore, HIV might reduce mBDNF by modifying the processing of proBDNF which, in turn, could promote axonal 
degeneration via a p75NTR-mediated mechanism. In this study, we used rodent neuronal cultures as well as postmortem brains of HIV-positive subjects to characterize whether proBDNF is involved in the primary mechanism of HIV-mediated neuronal loss. We observed a decrease in the levels of mBDNF accompanied by an increase in proBDNF in rat neurons exposed to gp120 as well as in the brain of HIV subjects. Our studies identify a new mechanism to explain HIV-mediated neurotoxicity and provide experimental data to suggest a novel therapeutic use of BDNF for the neurodegenerative pathology seen in HIV subjects.

\section{Materials and Methods}

Primary neuronal cultures. Cerebellar granule cells (CGCs) were prepared from 7-day-old Sprague Dawley rat pups as described previously (Bachis et al., 2006, 2009). Rat cortical neurons were prepared from embryonic day 17 (E17) embryos as described previously (Avdoshina et al., 2010). Cells were maintained at $37^{\circ} \mathrm{C}$ in $5 \% \mathrm{CO}_{2} / 95 \%$ air for $7-8 \mathrm{~d}$ before adding any compound.

Reagents. Human T-lymphotropic virus IIIB (HTLV-IIIB or HIV-1 ${ }_{\text {IIIB }}$ ) was obtained through the AIDS Research and Reference Reagent Program (from Dr. R. Gallo, Division of AIDS, NIAID, NIH) (Popovic et al., 1984; Ratner et al., 1985) and was used at a concentration of $1.5 \mathrm{ng} / \mathrm{ml}$ of p24. Gp120IIIB and tat were obtained from Immunodiagnostics and were used at a concentration of 5 and $100 \mathrm{~nm}$, respectively. proBDNF was purchased from Alomone Labs, and BDNF was a gift from Regeneron Pharmaceuticals. TAT-Pep5 was purchased from EMD4 Biosciences. p75NTR antibody (clone 192-IgG) was from Santa Cruz Biotechnology.

Neuronal processes. Cortical neurons were grown on coverslips, fixed, and stained with class III $\beta$-tubulin antibody (1:5000; Covance) at $4^{\circ} \mathrm{C}$. Coverslips were then incubated for $1 \mathrm{~h}$ at room temperature with the corresponding secondary antibody as described previously (Avdoshina et al., 2010). Cells were imaged using an FV300 laser confocal scanning system attached to an Olympus IX-70 upright microscope. The length of neuronal processes per coverslip was calculated on 25 neurons randomly selected by $2 \mathrm{D}$ Sholl analysis (Image J).

Cell survival. Cell survival was determined by Hoechst 33342/propidium iodide staining and 3(4,5-dimethylthiazol-2-yl)-2.5-diphenyltetrazolium bromide (MTT) assay. These analyses were performed as described previously (Bachis et al., 2003).

Human tissue. Brain tissues/sections of either sex were obtained from the National NeuroAIDS Tissue Consortium (NNTC) and the Section of Legal Medicine and Insurances of the University of Milan (Milan, Italy). Frozen tissue was homogenized using gentle sonication in lysis buffer composed of $1 \times$ Tris-buffered saline (TBS), 1\% NP-40, $1 \%$ Triton $\mathrm{X}-100,1 \mathrm{~mm}$ PMSF, $10 \%$ glycerol, and protease inhibitor cocktail (Sigma) on ice. The homogenates were incubated for 5 to $10 \mathrm{~min}$ on ice and then centrifuged at $14,000 \times g$ for $5 \mathrm{~min}$. Supernatants were collected and stored at $-80^{\circ} \mathrm{C}$. Total protein content was determined by Bradford Coomassie blue colorimetric assay.

ELISA. BDNF, nerve growth factor (NGF), and furin levels were determined using ELISA from Promega or R\&D, respectively, according to the manufacturer's instructions. The assay was performed as described previously (Nosheny et al., 2004). The ELISA for BDNF has a negligible cross reactivity with proBDNF $(\sim 2.5 \%)$ as determined by running in parallel a standard curve with proBDNF.

Western blot. Lysates were prepared by homogenization of human samples or rat neurons in lysis buffer as described above. Medium from cells was collected, centrifuged at 10,000 rpm for $10 \mathrm{~min}$ and concentrated using Centricon tubes. Media and lysate samples were subjected to immunoprecipitation. Samples were precleared using precipHen (Aves Labs) or protein A-Sepharose beads (Sigma), and the supernatant was then incubated with anti-BDNF Ab (Promega) or an anti-tPA Ab (Millipore) for $18 \mathrm{~h}$ at $4^{\circ} \mathrm{C}$. Immunoprecipitates were collected by centrifugation for $5 \mathrm{~min}$ at $5000 \mathrm{rpm}$. Beads were washed in lysis buffer and immune complexes were resolved by SDS-PAGE. Proteins were transferred onto a PVDF membrane and blocked with TBS-T ( $25 \mathrm{~mm}$ Tris and $1 \%$ Tween) containing 5\% milk powder. Blots were then incubated over-
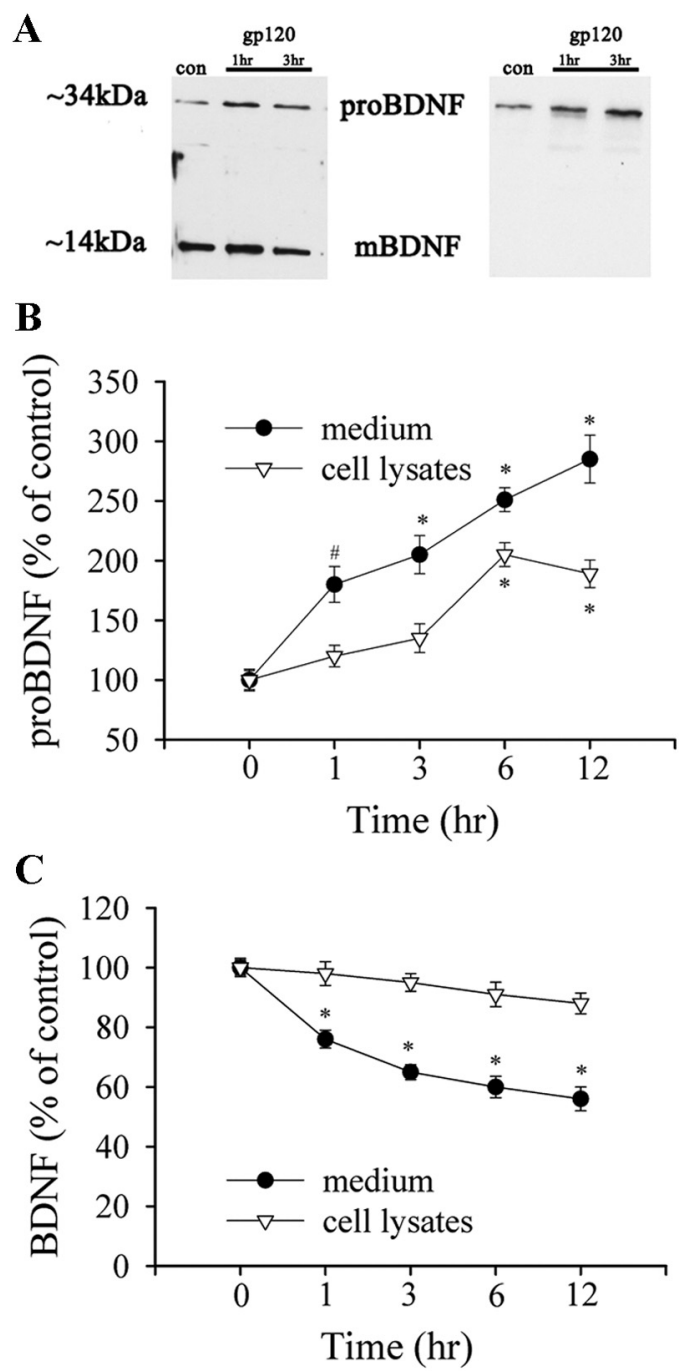

Figure 1. gp120 increases proBDNF. CGCs were exposed to gp120 for the indicated times, and levels of $\mathrm{mBDNF}$ and proBDNF were determined by ELISA and Western blot, respectively, in the medium and cell lysates. $A$, Representative Western blot analysis of CGC medium immunoprecipitated with an anti-BDNF antibody. The blot was analyzed with a BDNF antibody that recognizes either BDNF species (left) or proBDNF only (right). $\boldsymbol{B}$, Semiquantification of the 34 $\mathrm{kDa}$ band by densitometric analysis. $C$, Levels of BDNF as determined by ELISA. Data expressed as mean \pm SEM represent the average of three experiments ( $n=6$ each experiment). ${ }^{\#} p<0.05$, ${ }^{*} p<0.001$ vs control (ANOVA and Sheffe's test).

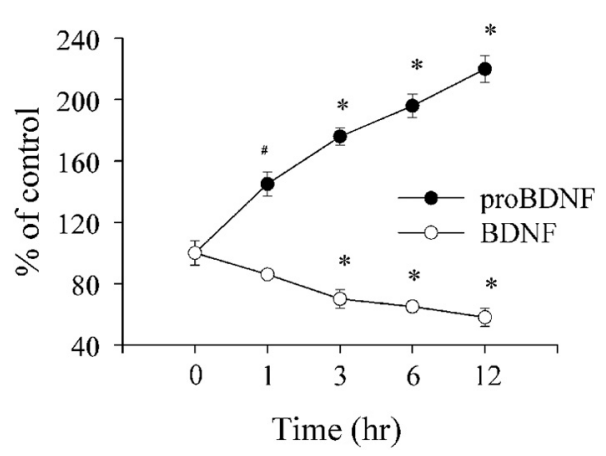

Figure 2. gp120 alters the release of mBDNF/proBDNF in cortical neurons. Cortical neurons, prepared as described previously (Avdoshina et al., 2010), were exposed to gp120 for the indicated times. The levels of mBDNF and proBDNF in the medium were determined by ELISA and Western blot, respectively, as described in Figure 1. Data are expressed as mean \pm SEM ( $n=6$ each time point). ${ }^{\#} p<0.05,{ }^{*} p<0.001$ vs control (ANOVA and Sheffe's test). 

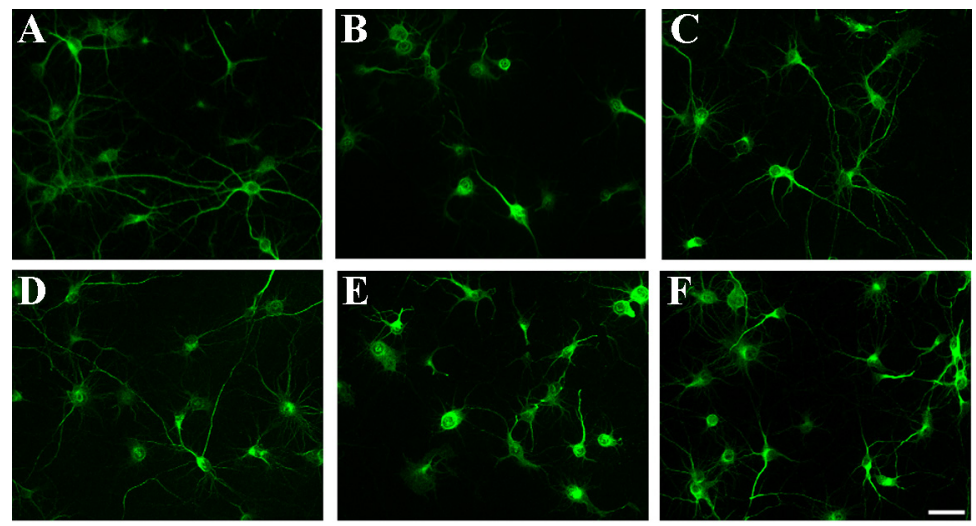

G

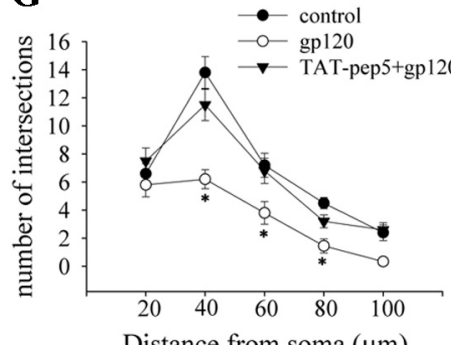

Distance from soma $(\mu \mathrm{m})$
H

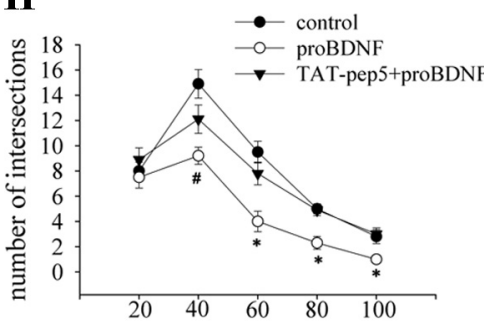

Distance from soma $(\mu \mathrm{m})$

Figure 3. gp120-induced neurite simplification is p75NTR dependent. $\boldsymbol{A}-\boldsymbol{F}$, Cortical neurons were exposed to boiled gp120 $(\boldsymbol{A})$ or medium $(\boldsymbol{D}), \mathrm{gp} 120(\boldsymbol{B})$, and proBDNF $(50 \mathrm{ng} / \mathrm{ml} ; \boldsymbol{E})$ alone or in combination with TAT-Pep5 $(100 \mathrm{~nm} ; \boldsymbol{C}, \boldsymbol{F})$ for $6 \mathrm{~h}$. Cells were then fixed and stained for class III $\beta$ tubulin. $\boldsymbol{G}, \boldsymbol{H}$, Quantification of neurite processes was then done as described in Materials and Methods. Data are the mean \pm SEM of 25 neurons. ${ }^{\#} p<0.05,{ }^{*} p<0.01$ vs control (ANOVA and Sheffe's test). Scale bar, $50 \mu \mathrm{m}$.

A

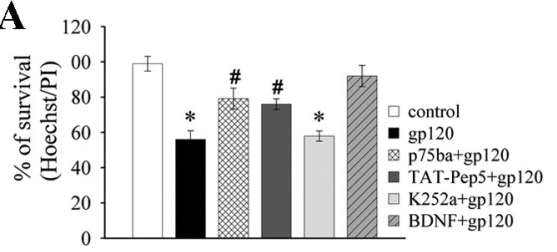

C

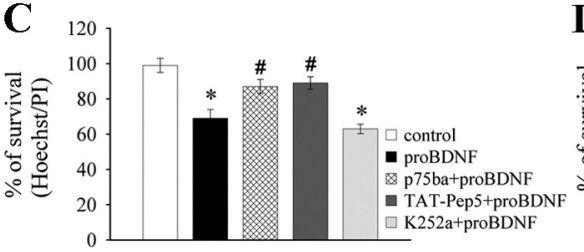

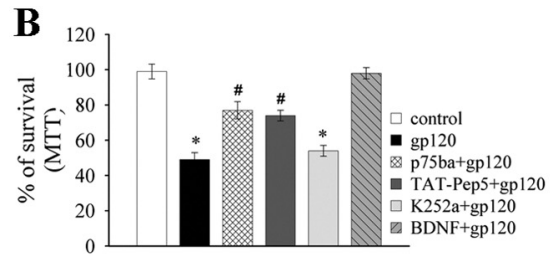

D

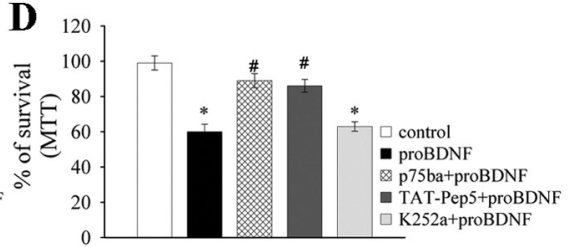

Figure 4. The toxic effect of gp 120 is p75NTR mediated. $A-D$, Cortical neurons were exposed to gp $120(\boldsymbol{A}, \boldsymbol{B})$ or proBDNF $(50 \mathrm{ng} / \mathrm{ml}$; $\boldsymbol{C}$, D) alone or in the presence of K252a (100 nM), TAT-Pep5 (100 nM), or p75ba (10 $\mu \mathrm{g} / \mathrm{ml})$. In $\boldsymbol{A}$ and $\boldsymbol{B}, \mathrm{BDNF}(50 \mathrm{ng} / \mathrm{ml})$ was added $3 \mathrm{~h}$ before gp120. Neuronal survival was determined $18 \mathrm{~h}$ later by cell count $(\boldsymbol{A}, \boldsymbol{C})$ or MTT $(\boldsymbol{B}, \boldsymbol{D})$ as described previously (Bachis et al., 2003). Data are expressed as the mean \pm SEM. $n=18 .{ }^{\#} p<0.05$ vs proBDNF or gp120; ${ }^{*} p<0.001$ vs control (ANOVA and Sheffe's test).

night with an anti-proBDNF Ab (dilution, 1:500; Alomone Labs) or an anti-BDNF antibody (dilution, 1:1000; Promega) or anti-tPA Ab (Millipore). After three washes with TBS-T, the blots were incubated with peroxidase-conjugated anti-rabbit secondary antibody (dilution, 1:1000; Santa Cruz Biotechnology) for $1 \mathrm{~h}$ at room temperature. Immunoreactivity was detected by enhanced chemiluminescence (Thermo Scientific).

Immunohistochemistry. Sections from the human cortex were obtained from the NNTC. After removal of paraffin, sections were incubated with $0.3 \% \mathrm{H}_{2} \mathrm{O}_{2}$ at room temperature to block endogenous peroxidase, and then rinsed and blocked in TBS with $0.25 \%$ Triton X-100 and $3 \%$ normal goat serum. Sections were incubated with a rabbit antiproBDNF antibody (Alomone Labs) overnight at $4^{\circ} \mathrm{C}$, rinsed in TBS, and incubated with a biotinylated anti-rabbit secondary antibody for $1 \mathrm{~h}$ at room temperature. Sections were then rinsed and incubated with $A B C$ Elite solution (1:400; Vector Labs) for $1 \mathrm{~h}$ at room temperature. The reaction was visualized by using TBS with $0.05 \% \quad 3,3^{\prime}$-diaminobenzidine and $0.01 \%$ $\mathrm{H}_{2} \mathrm{O}_{2}$. Sections were then rinsed, dehydrated, and coverslipped.

\section{Results}

Gp120 increases proBDNF in neurons

Postnatal rat CGCs in culture produce and release $\mathrm{mBDNF}$ in an activity-dependent manner (Marini et al., 1998), release proBDNF (Xu et al., 2011), and are sensitive to the neurotoxic action of both HIV and gp120 (Bachis et al., 2009). Thus, we first used these neurons to examine whether gp120 alters proBDNF processing.

CGCs were exposed to $5 \mathrm{~nm}$ gp120IIIB (gp120) for various time points. $\mathrm{mBDNF}$ and proBDNF were determined in the medium after immunoprecipitation with an antibody that recognizes both $\mathrm{mBDNF}(\sim 14 \mathrm{kDa})$ and proBDNF ( $\sim 34 \mathrm{kDa})$ species, followed by Western blot analysis. The medium of gp120treated CGCs contained less mBDNF but more proBDNF than control (Fig. 1A, left). The increase in proBDNF was confirmed with an antibody that recognizes proBDNF only (Fig. $1 A$, right). Time course analyses revealed that gp120 elicited a time-dependent accumulation of proBDNF in the medium starting at $1 \mathrm{~h}$ as well as in cell lysates by $6 \mathrm{~h}$ (Fig. $1 B$ ). Furthermore, mBDNF levels were determined by an ELISA that exhibits negligible ( $\sim 2.5 \%$ ) cross-reactivity with proBDNF. This assay confirmed that gp120 elicits a temporal decrease in mBDNF (Fig. 1C).

Cortical neurons in culture also release BDNF (Ghosh et al., 1994) and undergo apoptosis in the presence of HIV or gp120 (Bachis et al., 2009). Thus, we used these neurons to confirm that gp120 promotes the release of proBDNF. Cells were exposed to gp120 for various time points, and both proBDNF and mBDNF levels were determined in the medium. As for CGCs, gp120 induced an increase in proBDNF starting at $1 \mathrm{~h}$. This effect was followed by a decrease in $\mathrm{mBDNF}$ levels starting at $3 \mathrm{~h}$ (Fig. 2). Thus, it appears that gp120 affects the release of mBDNF and proBDNF in an opposite manner in different neuronal populations.

HIV, gp120, and proBDNF reduce the length of neuronal processes

Activation of p75NTR by proBDNF evokes apoptosis (Teng et al., 2005). CGCs and cortical neurons express both Trk and p75NTR (Courtney et al., 1997; Yaar et al., 1997). To examine the contribution of these receptors in the toxic action of gp120 through proBDNF, cortical neurons were exposed for various time points to gp120 or to a mutated form of proBDNF that cannot be cleaved into mBDNF (Koshimizu et al., 2009), in the presence or absence of TAT-Pep5, an intracellular inhibitor of p75NTR (Yamashita and Tohyama, 2003). Neurotoxicity was then determined by measuring the length of neuronal processes (axons and 

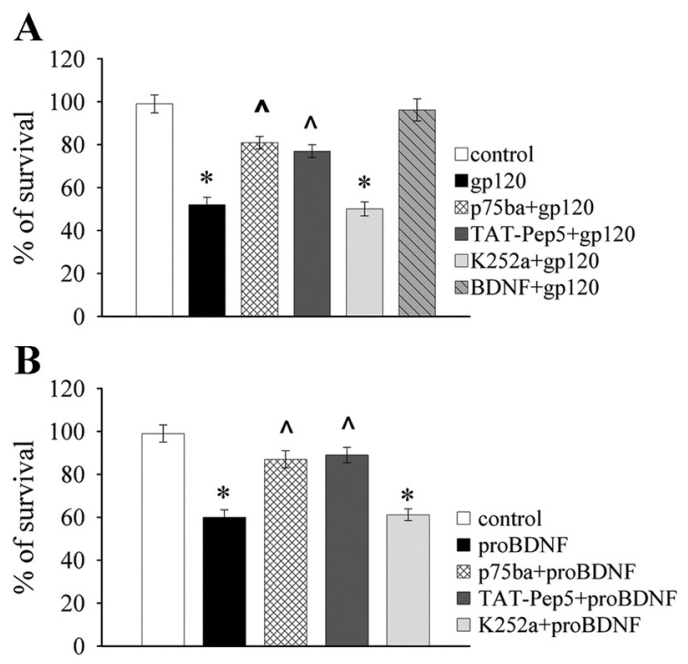

Figure 5. p75NTR-dependent reduction of neuronal survival. $A, B$, CGCS were exposed to gp120 $(\boldsymbol{A})$ or proBDNF $(50 \mathrm{ng} / \mathrm{ml} ; \boldsymbol{B})$ alone or in the presence of K252a (100 nM), TAT-Pep5 $(100$ $\mathrm{nm})$, or p $75 \mathrm{ba}(10 \mu \mathrm{g} / \mathrm{ml})$. In $A, B D N F(50 \mathrm{ng} / \mathrm{ml})$ was added $3 \mathrm{~h}$ before gp 120 . Neuronal survival was determined $18 \mathrm{~h}$ later as described in Figure 4 . Data are expressed as the mean \pm SEM. $n=$ 18. $\hat{p}<0.05$ vs proBDNF or gp120; ${ }^{*}<0.001$ vs control (ANOVA and Sheffe's test).

dendrites) as well as cell survival. An antibody against neuronspecific cytoskeletal protein class III $\beta$-tubulin was used to identify neuronal processes. Neurons exposed for $6 \mathrm{~h}$ to either gp 120 (Fig. $3 B$ ) or proBDNF (Fig. $3 E$ ) exhibited shorter neuronal processes than controls (Figs. $3 A, D, G, H$ ). The effect of gp120 was reproduced by HIV-IIIB but not by the trans-activator of transcription tat (data not shown), another viral protein that is neurotoxic (Eugenin et al., 2007). Shortening of neuronal processes mediated by gp120 and proBDNF was inhibited by TAT-Pep5 (Figs. $3 C, F-H$ ), suggesting that p75NTR activation plays a role in gp120 neurotoxicity.

To determine whether gp120-mediated pathology of synapses culminates in neuronal loss, cortical neurons were exposed to gp120 (Figs. $4 A, B$ ) or proBDNF (Fig. $4 C, D$ ) in the absence or presence of TAT-Pep5. Both proBDNF and gp120 promoted, by $18 \mathrm{~h}$, a significant decrease in neuronal survival as determined by neuronal counts (Figs. $4 A, C$ ) as well as MTT assay (Fig, $4 B, D$ ). TAT-Pep5 but not the Trk tyrosine kinase inhibitor K252a inhibited the toxic effect of both proBDNF and gp120 (Fig. 4), suggesting that the toxic effect of proBDNF occurs through the p75NTR (Teng et al., 2005). The results (Fig. 4) were confirmed by a p75NTR blocking antibody (p75ab). Nevertheless, TAT-Pep5 was less potent than recombinant BDNF in preventing gp120 toxicity (Fig. 4), supporting previous data that TrkB activation could also be a valid therapeutic tool to limit neuronal loss in HAD (Bachis et al., 2003).

We showed previously that CGCs are more sensitive to the toxic effect of gp120 than cortical neurons (Bachis et al., 2009). Therefore, we used CGCs to examine the reproducibility of the data obtained in cortical neurons. TAT-Pep 5 but not K252a prevented both gp120- and proBDNF-mediated neuronal loss (Fig. 5), further supporting the role of p75NTR in gp120 neurotoxicity.

\section{mBDNF and proBDNF levels in HIV subjects}

To establish whether HIV changes mBDNF/proBDNF levels in humans, we determined the content of $\mathrm{mBDNF}$ and proBDNF in postmortem human frontal cortex (CX), hippocampus (HP), and caudate/putamen (ST). Samples were from HIV-negative subjects, $\mathrm{HAD}$ subjects, $\mathrm{HIV}^{+}$subjects with normal neurocognitive diagnosis, and $\mathrm{HIV}^{+}$subjects with one or more opportunistic infections but no dementia (Table 1). We observed a reduction in the levels of $\mathrm{mBDNF}$ in HAD compared to HIV with no dementia in all brain areas examined by ELISA (Fig. $6 \mathrm{~A}$ ). We also determined the levels of another neurotrophin, NGF, in the same brain areas to examine the specificity of this finding. There was no significant difference in NGF levels between HAD versus non-HAD subjects (Fig. 6B).

To determine whether proBDNF levels are altered in HAD, we analyzed with Western blot the lysates from postmortem CX. In HAD subjects, the immunoreactivity corresponding to mBDNF decreased (Fig. 6C), supporting the data obtained with the ELISA, whereas the $34 \mathrm{kDA}$ immunoreactive band increased (Fig. 6C), suggesting an accumulation of proBDNF. The data were confirmed by using an antibody that recognizes proBDNF only. In fact, this antibody revealed an increase in the $34 \mathrm{kDa}$ species in HAD over HIV-negative individuals (Fig. 6D). The intensity of this band was increased in CX, ST, and HP of HAD subjects when compared to HIV subjects without dementia (Fig. 6E).

To establish which cells express proBDNF, sections from the CX of HIV-positive subjects with no dementia and HAD subjects were stained with the proBDNF antibody. ProBDNF immunoreactivity in non-HAD subjects was mainly localized in cell bodies and processes (Fig. 7A), suggesting a neuronal localization. Moreover, we observed an increase in the number of cells positive for proBDNF in HAD subjects (Fig. $7 B$ ) when compared to nonHAD subjects. In fact, cells positive for proBDNF per section were $22 \pm 3$ and $32 \pm 4$ SEM in control and HAD subjects, respectively $\left({ }^{*} p<0.05\right.$ vs control; $\left.n=3\right)$. Together, these data suggest that HIV reduces the processing of proBDNF. This event might contribute to synaptodendritic injury (Ellis et al., 2007) and synaptic dysfunction (McArthur et al., 2005) seen in HIVassociated neurocognitive disorders.

\section{Gp120 and HIV alter furin levels}

ProBDNF is cleaved by furin in the endoplasmic reticulum and Golgi to produce C-terminal mature neurotrophins (Mowla et al., 2001). Decreasing furin activity/synthesis may therefore have a central role in the ability of HIV/gp120 to increase proBDNF. To test this hypothesis, we determined intracellular levels of furin in CGCs exposed to gp120. We observed a time-dependent decrease in intracellular furin levels starting at $15 \mathrm{~min}$ (Fig. $8 \mathrm{~A}$ ). Thus, it appears that gp120 may increase proBDNF by reducing furin levels. To support this finding, we first analyzed furin content in brain tissue from HAD subjects. There was a decrease in furin in several areas of HAD brains (Fig. $8 \mathrm{~B}$ ). On the other hand, extracellular proBDNF is also proteolytically processed by plasmin to mBDNF. Plasminogen, the precursor to plasmin, is cleaved by tissue plasminogen activator (tPA), a protease that is also released from presynaptic vesicles. Therefore, we examined whether gp120 changes the levels of tPA. CGCs were exposed to gp120 for various time points. A decrease in tPA levels was observed only starting at $3 \mathrm{~h}$ after gp 120 (Figs. $8 C, D$ ). Therefore, this temporal delay on tPA cannot explain the gp120-mediated accumulation of proBDNF which occurs within $1 \mathrm{~h}$.

\section{Discussion}

The severity of cognitive impairment in HIV-positive subjects correlates with synaptodendritic degeneration (Ellis et al., 2007). The present work was undertaken to provide insight into novel molecular mechanisms by which HIV promotes synaptic simplification. Here we show that neurons exposed to gp120 exhibit 
Table 1. Characteristics of study samples

\begin{tabular}{|c|c|c|c|c|c|c|c|c|}
\hline $\begin{array}{l}\text { Sample } \\
\text { number }\end{array}$ & HIV-1 status & Age & Gender & ARV use & Drug abuse & CNS pathologies & Neurocognitive diagnosis & $\begin{array}{l}\text { CSF VL } \\
\text { (copies/ml) }\end{array}$ \\
\hline 1 & + & 34 & $\mathrm{~F}$ & $Y$ & None & Hypoxic/ischemic damage & $\mathrm{HAD}$ & 76 \\
\hline 2 & + & 57 & M & N & None & Atherosclerosis of the brain & HAD & 2747 \\
\hline 3 & + & 53 & M & $Y$ & None & NA & HAD & 50 \\
\hline 4 & + & 40 & $M$ & $\mathrm{~N}$ & None & NA & $\mathrm{HAD}$ & 400 \\
\hline 5 & + & 34 & M & $Y$ & NA & Minimal abnormalities & $\mathrm{HAD}$ & NA \\
\hline 6 & + & 34 & $M$ & $\mathrm{~N}$ & None & Minimal abnormalities & $\mathrm{HAD}$ & NA \\
\hline 7 & + & 40 & $M$ & $\mathrm{~N}$ & NA & Minimal abnormalities & HAD & NA \\
\hline 8 & + & 35 & M & Y & NA & Minimal abnormalities & HAD & NA \\
\hline 9 & + & 30 & M & $Y$ & None & Microglial nodule encephalitis & CMV encephalitis & 9627 \\
\hline 10 & + & 43 & $\mathrm{~F}$ & Y & Past cocaine, opiate & Bacterial parenchymal infection, contusion & Neuropsychological impairment due to other causes & 50 \\
\hline 11 & + & 61 & M & NA & NA & CMV encephalitis, Optic nerve atrophy & Neuropsychological impairment due to other causes & 2617 \\
\hline 12 & + & 46 & $\mathrm{~F}$ & Y & Cocaine & No known pathology & Neuropsychological impairment due to other causes & 27400 \\
\hline 13 & + & 35 & M & $Y$ & NA & CMV encephalitis, aseptic leptomeningitis & Neuropsychological impairment due to other causes & 50 \\
\hline 14 & + & 49 & M & Y & None & CMV encephalitis, microglial nodule encephalitis & Neuropsychological impairment due to other causes & 841 \\
\hline 15 & + & 44 & M & Y & Past cocaine & CMV encephalitis, focal infarct, HIVE & Neuropsychological impairment due to other causes & 100000 \\
\hline 16 & + & 46 & M & Y & Past cocaine & No known pathology & Neurocognitive normal & 2355 \\
\hline 17 & + & 45 & M & Y & None & Focal infarct, contusion & Neurocognitive normal & 11405 \\
\hline 18 & + & 51 & M & NA & Past cocaine & Bacterial parenchymal infection & Neurocognitive normal & 53215 \\
\hline 19 & + & 37 & M & Y & NA & Cryptococcus & Neurocognitive normal & 400 \\
\hline 20 & + & 47 & M & $Y$ & None & Other noninfectious path & Neurocognitive normal & NA \\
\hline 21 & + & 34 & $\mathrm{~F}$ & $Y$ & NA & Other noninfectious path & Neurocognitive normal & 50 \\
\hline 22 & + & 39 & M & $Y$ & NA & Minimal abnormalities & Neurocognitive normal & NA \\
\hline 23 & - & 18 & M & None & None & NA & Neurocognitive normal & None \\
\hline 24 & - & 37 & M & None & None & NA & Neurocognitive normal & None \\
\hline 25 & - & 52 & M & None & None & NA & Neurocognitive normal & None \\
\hline
\end{tabular}

F, Female; M, male; Y, yes; N, no; NA, not available; CMV, cytomegalovirus; VL, viral load; ARV, antiretroviral; HIVE, HIV encephalitis.

A

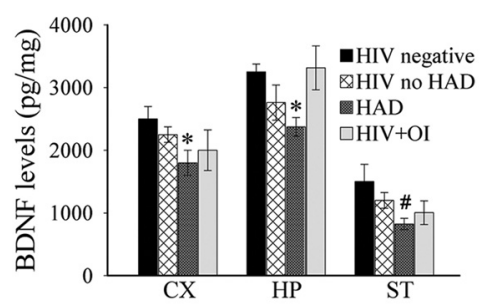

C

D

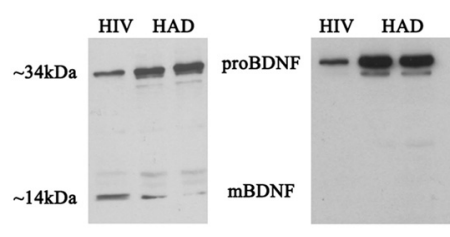

B

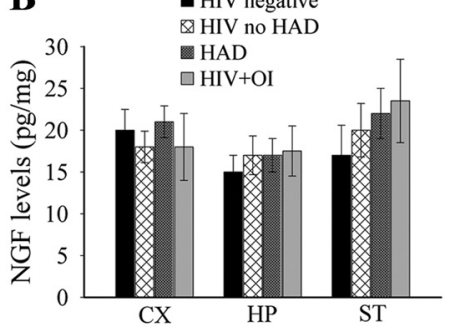

E

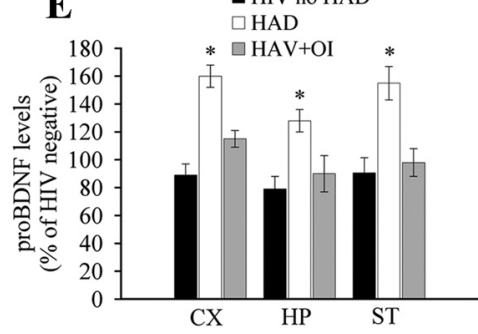

Figure 6. BDNF levels are decreased in the brain of HAD subjects. $\boldsymbol{A}, \boldsymbol{B}, \operatorname{BDNF}(\boldsymbol{A})$ or NGF $(\boldsymbol{B})$ levels were measured by ELISA in human $C X, H P$, and ST. Samples were from HIV-negative subjects, HIV-positive subjects with normal neurocognitive diagnosis (HIV but not HAD), HIV-positive subjects with HAD, and HIV-positive subjects with one or more opportunistic infections, such as encephalitis [HIV + Ol (opportunistic infections)]. Data are the mean \pm SEM of subjects described in Table $1 .{ }^{*} p<0.005 ;{ }^{\#} p<$ 0.01 vs control (ANOVA and Sheffe's test). C, D, Representative Western blots of cortical lysates from HIV and HAD subjects analyzed with an anti-BDNF antibody or an anti-proBDNF antibody, respectively. $\boldsymbol{E}$, Relative levels of proBDNF in the indicated areas as determined by densitometric analysis of the $34 \mathrm{kDa}$ band. Data are expressed as the mean $\pm \operatorname{SEM}(n=7) .{ }^{*} p<0.05$ vs HIV but not HAD (ANOVA and Sheffe's test).

lower concentrations of mBDNF and higher levels of proBDNF than controls. Most importantly, these data were reproduced in human subjects. In fact, proBDNF levels in HAD subjects were higher than those in HIV-negative as well as HIV-positive subjects without dementia. mBDNF plays a key role in axonal branching, whereas proBDNF reduces synaptic plasticity (for re-

view, see Greenberg et al., 2009). Thus, the altered $\mathrm{mBDNF} /$ proBDNF ratio in HIV subjects could compromise synaptic connections and neuronal survival. Overall, our data suggest that the neurotoxic effects of HIV may encompass a reduction of the neurotrophic factor environment. Understanding how HIV inhibits the availability of mBDNF is crucial for the development of new therapies.

Most of the neurotoxic properties of HIV have been attributed to the combined effect of the virus and viral proteins, and/or host immune responses (Kaul et al., 2001). In this study we show that HIVinfected individuals with HAD exhibit lower BDNF levels than nondemented $\mathrm{HIV}$-positive individuals in the CX, ST, and HP. BDNF is made in the cerebral cortex and delivered to the striatal neurons where it is particularly important for their survival and for the activity of the corticostriatal synapses (Zuccato and Cattaneo, 2007). Conversely, loss of BDNF has been suggested to be a risk factor in chronic diseases of the basal ganglia such as Parkinson's (Nagatsu et al., 2000) and Huntington's diseases (Zuccato et al., 2001). BDNF is also abundant in the HP, where it is important in maintaining dendritic morphology and synaptic function (Horch and Katz, 2002), as well as the survival of neurons and their connections (Xu et al., 2000). Indeed, evidence has shown a strong correlation between reduction of BDNF and decrease in hippocampal neuronal survival and mem- 

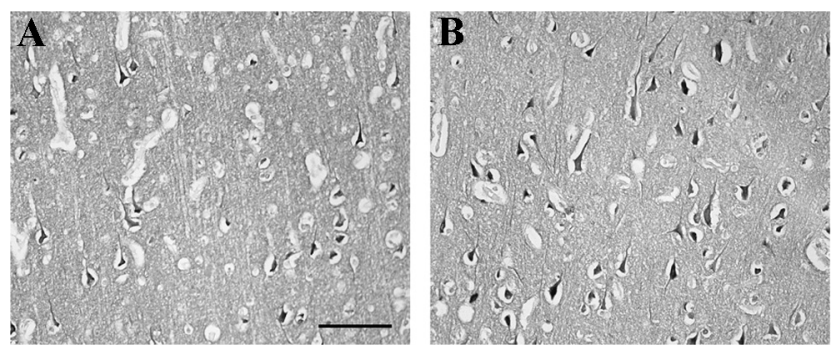

Figure 7. Neuronal localization of proBDNF in human brains. $\boldsymbol{A}, \boldsymbol{B}$, Sections from the cortex of HIV-positive subjects without $(\boldsymbol{A})$ and with $\operatorname{HAD}(\boldsymbol{B})$ were processed as described in Materials and Methods. Sections were incubated with a rabbit anti-proBDNF antibody (Alomone Labs), rinsed in TBS, and incubated with a biotinylated anti-rabbit secondary antibody. Sections were then rinsed and incubated with $A B C$ Elite solution. The reaction was visualized by using TBS with $0.05 \% 3,3^{\prime}$-diaminobenzidine and $0.01 \% \mathrm{H}_{2} \mathrm{O}_{2}$. Sections were then rinsed, dehydrated, and coverslipped. Scale bar, $200 \mu \mathrm{m}$. Note the higher number of proBDNF-positive neurons in the HAD section.

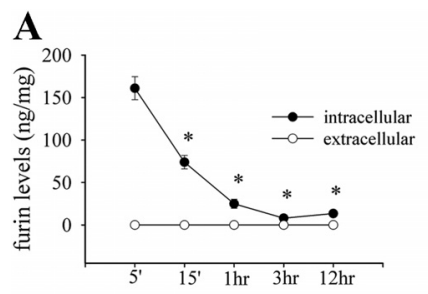

C

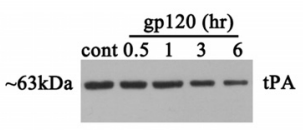

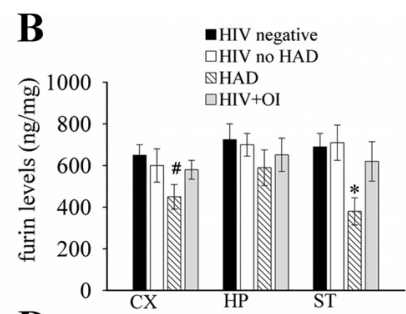

D

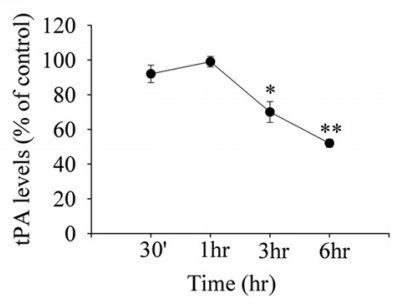

Figure 8. HIV and gp120 decrease furin levels. $\boldsymbol{A}$, Intracellular and extracellular furin levels were measured by ELISA in cortical neurons exposed to gp 120 for the indicated times. Data are the means \pm SEM of six samples ( ${ }^{*} p<0.001$ vs control, ANOVA and Sheffe's test). $\boldsymbol{B}$, Furin levels were measured in the indicated human brain areas by ELISA. Data are the means \pm SEM of five samples. ${ }^{\#} p<0.05 ;{ }^{*} p<0.001$ vs HIV negative (ANOVA and Sheffe's test). C, Example of a Western blot analysis of medium of neurons exposed to gp120 for the indicated times. $\boldsymbol{D}$, Relative levels of tPA in the medium as determined by densitometric analysis of a $63 \mathrm{kDa}$ band. Data expressed as mean \pm SEM represent the average of three experiments ( $n=4$ in each experiment). ${ }^{*} p<0.05,{ }^{* *} p<0.001$ vs control (ANOVA and Sheffe's test).

ory (Erickson et al., 2010). Pathological features consistent with lack of BDNF in these brain areas have also been described in HAD subjects. In fact, cortical neurons of HAD subjects are characterized by axonal injury (Ellis et al., 2007) as well as apoptosis (Garden et al., 2002). Hippocampal dysfunction has also been described in HIV-positive women (Maki et al., 2009). In addition, HIV promotes pathological changes in the basal ganglia. Abnormalities include neuronal loss in the putamen (Everall et al., 1995) and globus pallidus (Fox et al., 1997), loss of nigrostriatal dopamine neurons (Reyes et al., 1991; Itoh et al., 2000), and dysfunctional dopaminergic transport (Wang et al., 2004). These correlations allow us to speculate that the decrease in BDNF evoked by HIV contributes to the development of synaptic simplification and neuronal damage seen in HAD.

An important finding reported here is the effect of HIV and its soluble envelope protein gp 120 on proBDNF. ProBDNF was detected in the medium of rodent neurons, consistent with the notion that mature neurons are capable of producing and releasing proBDNF (Peng et al., 2005; Yang et al., 2009b). Secreted proBDNF has an opposite effect of $\mathrm{mBDNF}$ on neuronal plasticity (Pang et al., 2004; Woo et al., 2005). In fact, BDNF promotes neuronal survival and maintenance of synaptic spines through the high-affinity receptor TrkB (Dorsey et al., 2006), whereas through sortilin, proBDNF binds to p75NTR and induces apoptosis (Teng et al., 2005). We have observed that proBDNF reduces the survival of cortical neurons and CGCs. In addition, proBDNF reduced the length of neuronal processes in these neuronal cultures. These toxic properties of proBDNF were inhibited by blocking p75NTR activity, either by TAT-Pep5 or by a p75ab. On the contrary, K252a, an inhibitor of Trk signaling (Berg et al., 1992; Ohmichi et al., 1992) failed to reverse proBDNF toxicity. In contrast, BDNF prevented gp120 activity, confirming previous results (Bachis et al., 2003). Thus, our data support the notion that $\mathrm{mBDNF}$ and proBDNF elicit opposite effects through the activation of two distinct receptors, Trk and p75NTR. Most importantly, a similar neurotoxic profile was elicited by gp120 (or HIV). In fact, TAT-Pep5 but not K252a reduced significantly the ability of gp120 to promote neuronal injury. Intriguingly, whereas TAT-Pep 5 inhibited the ability of gp120 to reduce the length of neuronal processes, this p75NTR antagonist could not completely reverse gp 120-mediated cell death. This could be due a number of mechanisms. For instance, two studies have showed that the DR6 receptor, which like p75NTR is a member of the tumor necrosis factor receptor family, causes axonal degeneration (Nikolaev et al., 2009; Park et al., 2010). Thus, a blockade of p75NTR may be insufficient to fully protect against gp120 because it does not prevent DR6 activation. In addition, the loss of BDNF combined with an increase of proinflammatory cytokines (Medders et al., 2010) might exacerbate the neurotoxic profile of gp120. Thus, we cannot exclude that gp120 promotes cell death via more than one mechanism. Overall, our data support the hypothesis that HIV, most likely through gp120 (Bachis et al., 2009 ), causes a change in the ratio of proBDNF to mBDNF that ultimately results in an increased release of proBDNF. This altered ratio promotes an environment that is conducive to activation of p75NTR. This could be a risk factor for the development of synaptic simplifications and neuronal apoptosis seen in HAD.

ProBDNF can be converted into mBDNF intracellularly in the trans-Golgi by endoproteases such as furin or in the immature secretory granules by proprotein convertases (Mowla et al., 2001). However, proBDNF can also be cleaved to mBDNF extracellularly by proteases including tPA. Reduced tPA release has been shown to enhance proBDNF signaling through p75NTR, leading to loss of spines and synapses and subsequently neuronal loss (Head et al., 2009). Therefore, we examined the levels of tPA and furin to reveal the molecular mechanisms underlying the effect of HIV on proBDNF processing. Our data show that gp120 reduces the release of tPA. However, this reduction does not temporally correlate with the gp120-mediated increase in proBDNF, which occurs as early as $15 \mathrm{~min}$ after exposure of neurons to the envelope protein. Contrary to tPA, furin levels were reduced as early as $15 \mathrm{~min}$ after gp 120 , suggesting that furin is a key enzyme in gp120-mediated effect on proBDNF. How gp120 reduces furin levels is still under investigation. Furin mRNA levels did not change up to $3 \mathrm{~h}$ (data not shown), suggesting that gp120 may not affect furin transcription. On the other hand, furin has been shown to localize in early endosomes to be recycled to cell surface or trafficked to the trans-Golgi network (Molloy et al., 1998). Gp120 is internalized within minutes by axons and transported in endosomes (Bachis et al., 2006). Thus, 
gp120 might change furin trafficking and help promote furin degradation, perhaps through ubiquitination. This could be consistent with the rapid ( 15 min) effect of gp120 on furin and the consequent increase in the levels of unprocessed (within $1 \mathrm{~h}$ ) proBDNF. Regulation of furin activity/synthesis might therefore have a central role in determining which neurons die even if they are not productively infected. However, this hypothesis must be proven.

In conclusion, our data suggest that gp120 shed by the virus can inhibit the appropriate processing of proBDNF into mature BDNF by reducing furin levels. proBDNF, in turn, initiates neuronal damage which, in combination with other neurotoxins such as glutamate or $\mathrm{TNF} \alpha$, can lead to apoptosis and neuronal loss. Our data provide support for new pharmacological agents against HIV-mediated neuronal toxicity that are able to increase mBDNF as well as promote the processing of proBDNF or inhibit proBDNF activity.

\section{References}

Avdoshina V, Biggio F, Palchik G, Campbell LA, Mocchetti I (2010) Morphine induces the release of CCL5 from astrocytes: potential neuroprotective mechanism against the HIV protein gp120. Glia 58:1630-1639.

Avdoshina V, Garzino-Demo A, Bachis A, Monaco MC, Maki PM, Tractenberg RE, Liu C, Young MA, Mocchetti I (2011) HIV-1 decreases the levels of neurotrophins in human lymphocytes. AIDS 25:1126-1128.

Bachis A, Major EO, Mocchetti I (2003) Brain-derived neurotrophic factor inhibits human immunodeficiency virus-1/gp120-mediated cerebellar granule cell death by preventing gp120 internalization. J Neurosci 23:5715-5722.

Bachis A, Aden SA, Nosheny RL, Andrews PM, Mocchetti I (2006) Axonal transport of human immunodeficiency virus type 1 envelope glycoprotein 120 is found in association with neuronal apoptosis. J Neurosci 26:6771-6780.

Bachis A, Biggio F, Major EO, Mocchetti I (2009) M- and T-tropic HIVs promote apoptosis in rat neurons. J Neuroimmune Pharmacol 4:150-160.

Berg MM, Sternberg DW, Parada LF, Chao MV (1992) K-252a inhibits nerve growth factor-induced trk proto-oncogene tyrosine phosphorylation and kinase activity. J Biol Chem 267:13-16.

Conant K, McArthur JC, Griffin DE, Sjulson L, Wahl LM, Irani DN (1999) Cerebrospinal fluid levels of MMP-2, 7, and 9 are elevated in association with human immunodeficiency virus dementia. Ann Neurol 46:391-398.

Courtney MJ, Akerman KE, Coffey ET (1997) Neurotrophins protect cultured cerebellar granule neurons against the early phase of cell death by a two-component mechanism. J Neurosci 17:4201-4211.

Dorsey SG, Renn CL, Carim-Todd L, Barrick CA, Bambrick L, Krueger BK, Ward CW, Tessarollo L (2006) In vivo restoration of physiological levels of truncated TrkB.T1 receptor rescues neuronal cell death in a trisomic mouse model. Neuron 51:21-28.

Ellis R, Langford D, Masliah E (2007) HIV and antiretroviral therapy in the brain: neuronal injury and repair. Nat Rev Neurosci 8:33-44.

Erickson KI, Prakash RS, Voss MW, Chaddock L, Heo S, McLaren M, Pence BD, Martin SA, Vieira VJ, Woods JA, McAuley E, Kramer AF (2010) Brain-derived neurotrophic factor is associated with age-related decline in hippocampal volume. J Neurosci 30:5368-5375.

Eugenin EA, King JE, Nath A, Calderon TM, Zukin RS, Bennett MV, Berman JW (2007) HIV-tat induces formation of an LRP-PSD-95-NMDARnNOS complex that promotes apoptosis in neurons and astrocytes. Proc Natl Acad Sci U S A 104:3438-3443.

Everall IP, Hudson L, al-Sarraj S, Honavar M, Lantos P, Kerwin R (1995) Decreased expression of AMPA receptor messenger RNA and protein in AIDS: a model for HIV-associated neurotoxicity. Nat Med 1:1174-1178.

Fox L, Alford M, Achim C, Mallory M, Masliah E (1997) Neurodegeneration of somatostatin-immunoreactive neurons in HIV encephalitis. J Neuropathol Exp Neurol 56:360-368.

Garden GA, Budd SL, Tsai E, Hanson L, Kaul M, D’Emilia DM, Friedlander RM, Yuan J, Masliah E, Lipton SA (2002) Caspase cascades in human immunodeficiency virus-associated neurodegeneration. J Neurosci 22:4015-4024.
Gartner S, Liu Y (2002) Insights into the role of immune activation in HIV neuropathogenesis. J Neurovirol 8:69-75.

Ghosh A, Carnahan J, Greenberg ME (1994) Requirement for BDNF in activity-dependent survival of cortical neurons. Science 263:1618-1623.

Greenberg ME, Xu B, Lu B, Hempstead BL (2009) New insights in the biology of BDNF synthesis and release: implications in CNS function. J Neurosci 29:12764-12767.

Head BP, Patel HH, Niesman IR, Drummond JC, Roth DM, Patel PM (2009) Inhibition of p75 neurotrophin receptor attenuates isoflurane-mediated neuronal apoptosis in the neonatal central nervous system. Anesthesiology 110:813-825.

Horch HW (2004) Local effects of BDNF on dendritic growth. Rev Neurosci 15:117-129.

Horch HW, Katz LC (2002) BDNF release from single cells elicits local dendritic growth in nearby neurons. Nat Neurosci 5:1177-1184.

Itoh K, Mehraein P, Weis S (2000) Neuronal damage of the substantia nigra in HIV-1 infected brains. Acta Neuropathol (Berl) 99:376-384.

Kaul M, Garden GA, Lipton SA (2001) Pathways to neuronal injury and apoptosis in HIV-associated dementia. Nature 410:988-994.

Kerr SJ, Armati PJ, Pemberton LA, Smythe G, Tattam B, Brew BJ (1997) Kynurenine pathway inhibition reduces neurotoxicity of HIV-1-infected macrophages. Neurology 49:1671-1681.

Koshimizu H, Kiyosue K, Hara T, Hazama S, Suzuki S, Uegaki K, Nagappan G, Zaitsev E, Hirokawa T, Tatsu Y, Ogura A, Lu B, Kojima M (2009) Multiple functions of precursor BDNF to CNS neurons: negative regulation of neurite growth, spine formation and cell survival. Mol Brain 2:27.

Li Y, Luikart BW, Birnbaum S, Chen J, Kwon CH, Kernie SG, Bassel-Duby R, Parada LF (2008) TrkB regulates hippocampal neurogenesis and governs sensitivity to antidepressive treatment. Neuron 59:399-412.

Maki PM, Cohen MH, Weber K, Little DM, Fornelli D, Rubin LH, Perschler P, Gould F, Martin E (2009) Impairments in memory and hippocampal function in HIV-positive vs HIV-negative women. Neurology 72:1661-1668.

Marini AM, Rabin SJ, Lipsky RH, Mocchetti I (1998) Activity-dependent release of brain-derived neurotrophic factor underlies the neuroprotective effect of $N$-methyl-D-aspartate. J Biol Chem 273:29394-29399.

Masliah E (1995) Mechanisms of synaptic dysfunction in Alzheimer's disease. Histol Histopathol 10:509-519.

McArthur JC, Brew BJ, Nath A (2005) Neurological complications of HIV infection. Lancet Neurol 4:543-555.

Medders KE, Sejbuk NE, Maung R, Desai MK, Kaul M (2010) Activation of p38 MAPK is required in monocytic and neuronal cells for HIV glycoprotein 120-induced neurotoxicity. J Immunol 185:4883-4895.

Molloy SS, Thomas L, Kamibayashi C, Mumby MC, Thomas G (1998) Regulation of endosome sorting by a specific PP2A isoform. J Cell Biol 142:1399-1411.

Mowla SJ, Farhadi HF, Pareek S, Atwal JK, Morris SJ, Seidah NG, Murphy RA (2001) Biosynthesis and post-translational processing of the precursor to brain-derived neurotrophic factor. J Biol Chem 276:12660-12666.

Nagatsu T, Mogi M, Ichinose H, Togari A (2000) Changes in cytokines and neurotrophins in Parkinson's disease. J Neural Transm Suppl 60:277-290.

Nikolaev A, McLaughlin T, O'Leary DD, Tessier-Lavigne M (2009) APP binds DR6 to trigger axon pruning and neuron death via distinct caspases. Nature 457:981-989.

Nosheny RL, Bachis A, Acquas E, Mocchetti I (2004) Human immunodeficiency virus type 1 glycoprotein gp120 reduces the levels of brain-derived neurotrophic factor in vivo: potential implication for neuronal cell death. Eur J Neurosci 20:2857-2864.

Ohmichi M, Decker SJ, Pang L, Saltiel AR (1992) Inhibition of the cellular actions of nerve growth factor by staurosporine and K252A results from the attenuation of the activity of the trk tyrosine kinase. Biochemistry 31:4034-4039.

Pang PT, Teng HK, Zaitsev E, Woo NT, Sakata K, Zhen S, Teng KK, Yung WH, Hempstead BL, Lu B (2004) Cleavage of proBDNF by tPA/plasmin is essential for long-term hippocampal plasticity. Science 306:487-491.

Park KJ, Grosso CA, Aubert I, Kaplan DR, Miller FD (2010) p75NTRdependent, myelin-mediated axonal degeneration regulates neural connectivity in the adult brain. Nat Neurosci 13:559-566.

Peng S, Wuu J, Mufson EJ, Fahnestock M (2005) Precursor form of brainderived neurotrophic factor and mature brain-derived neurotrophic fac- 
tor are decreased in the pre-clinical stages of Alzheimer's disease. J Neurochem 93:1412-1421.

Popovic M, Sarngadharan MG, Read E, Gallo RC (1984) Detection, isolation, and continuous production of cytopathic retroviruses (HTLV-III) from patients with AIDS and pre-AIDS. Science 224:497-500.

Raff MC, Whitmore AV, Finn JT (2002) Axonal self-destruction and neurodegeneration. Science 296:868-871.

Ratner L, Haseltine W, Patarca R, Livak KJ, Starcich B, Josephs SF, Doran ER, Rafalski JA, Whitehorn EA, Baumeister K, Ivanoff L, Petteway SR, Pearson ML, Lautenberger JA, Papas TS, Ghrayeb J, Chang NT, Gallo RC, Wong-Staal F (1985) Complete nucleotide sequence of the AIDS virus, HTLV-III. Nature 313:277-284.

Reyes MG, Faraldi F, Senseng CS, Flowers C, Fariello R (1991) Nigral degeneration in acquired immune deficiency syndrome (AIDS). Acta Neuropathol (Berl) 82:39-44.

Tanaka J, Horiike Y, Matsuzaki M, Miyazaki T, Ellis-Davies GC, Kasai H (2008) Protein synthesis and neurotrophin-dependent structural plasticity of single dendritic spines. Science 319:1683-1687.

Teng HK, Teng KK, Lee R, Wright S, Tevar S, Almeida RD, Kermani P, Torkin R, Chen ZY, Lee FS, Kraemer RT, Nykjaer A, Hempstead BL (2005) ProBDNF induces neuronal apoptosis via activation of a receptor complex of p75NTR and sortilin. J Neurosci 25:5455-5463.

Wang GJ, Chang L, Volkow ND, Telang F, Logan J, Ernst T, Fowler JS (2004) Decreased brain dopaminergic transporters in HIV-associated dementia patients. Brain 127:2452-2458.

Woo NH, Teng HK, Siao CJ, Chiaruttini C, Pang PT, Milner TA, Hempstead
BL, Lu B (2005) Activation of p75NTR by proBDNF facilitates hippocampal long-term depression. Nat Neurosci 8:1069-1077.

Xu B, Zang K, Ruff NL, Zhang YA, McConnell SK, Stryker MP, Reichardt LF (2000) Cortical degeneration in the absence of neurotrophin signaling: dendritic retraction and neuronal loss after removal of the receptor TrkB. Neuron 26:233-245.

Xu ZQ, Sun Y, Li HY, Lim Y, Zhong JH, Zhou XF (2011) Endogenous proBDNF is a negative regulator of migration of cerebellar granule cells in neonatal mice. Eur J Neurosci 33:1376-1384.

Yaar M, Zhai S, Pilch PF, Doyle SM, Eisenhauer PB, Fine RE, Gilchrest BA (1997) Binding of beta-amyloid to the p75 neurotrophin receptor induces apoptosis. A possible mechanism for Alzheimer's disease. J Clin Invest 100:2333-2340.

Yamashita T, Tohyama M (2003) The p75 receptor acts as a displacement factor that releases Rho from Rho-GDI. Nat Neurosci 6:461-467.

Yang F, Je HS, Ji Y, Nagappan G, Hempstead B, Lu B (2009a) Pro-BDNFinduced synaptic depression and retraction at developing neuromuscular synapses. J Cell Biol 185:727-741.

Yang J, Siao CJ, Nagappan G, Marinic T, Jing D, McGrath K, Chen ZY, Mark W, Tessarollo L, Lee FS, Lu B, Hempstead BL (2009b) Neuronal release of proBDNF. Nat Neurosci 12:113-115.

Zuccato C, Cattaneo E (2007) Role of brain-derived neurotrophic factor in Huntington's disease. Prog Neurobiol 81:294-330.

Zuccato C, Ciammola A, Rigamonti D, Leavitt BR, Goffredo D, Conti L, MacDonald ME, Friedlander RM, Silani V, Hayden MR, Timmusk T, Sipione S, Cattaneo E (2001) Loss of Huntingtin-mediated BDNF gene transcription in Huntington's disease. Science 293:493-498. 